\title{
GENOMICS
}

\section{Inferring tumor subclonality}

\author{
A framework for evaluating tools that reconstruct tumor subclonal structure.
}

1 umor samples consist of subclones with different mutations and evolutionary trajectories. Inference of tumor subclonality using DNA sequencing data is challenging. "There are a remarkable number of methods for doing this, and effectively no good benchmarks of which work well and which do not," says Paul Boutros, from the University of Toronto and the University of California, Los Angeles.

Boutros shared the idea of building a framework for benchmarking subclonal reconstruction methods with others, which led to a community undertaking. Inferring tumor subclonality needs to estimate global characteristics of tumor composition, assign individual mutations to each subclone, and reconstruct phylogenetic relationships. For each task, the team assessed a set of metrics and selected the most suitable ones.
Although this sounds straightforward, Boutros was surprised by how much experts sometimes disagreed. "In one part of the study we showed experts in the field a true evolutionary history for a tumor, and then several different types of mistakes that might be made in reconstructing it. We then asked them, 'Which mistake is worst?', and while there was agreement in many situations, in others experts really prioritized different aspects of tumor evolution." This is where a community-based approach helped greatly.

There are other components of the framework, including a simulator of tumor genomes and a tool for efficiently executing a library of methods for subclonal reconstruction. Using these resources, Boutros and colleagues found the performance of a method is often influenced by factors such as read depth and the detection pipeline for single nucleotide variants. The framework is being used in the ICGC-TCGA DREAM Somatic Mutation Calling Tumor Heterogeneity Challenge for evaluating subclonal reconstruction methods. Beyond that, "I hope that people will use this strategy to develop more tightly tailored benchmarks for specific biological situations of interest not touched in our work (e.g., comparing primary and metastatic disease)," says Boutros.

\section{Lin Tang}

Published online: 4 March 2020

https://doi.org/10.1038/s41592-020-0777-0

Research paper

Salcedo, A. et al. A community effort to create standards for evaluating tumor subclonal reconstruction. Nat. Biotechnol. 37, 1163-1173 (2019).

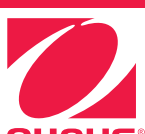
OHAUS
Versatile high-throughput Bead Mill Homogenizer is ideal for processing multiple tubes, microplates, deep-well plates, or any sample container that fits in the open-platform sample holder.

\section{Precision and Analytical} Lab Balances with large weighing surfaces, a space-saving draft shield design, and full housing in-use cover allow for use in lab, education and industrial environments.

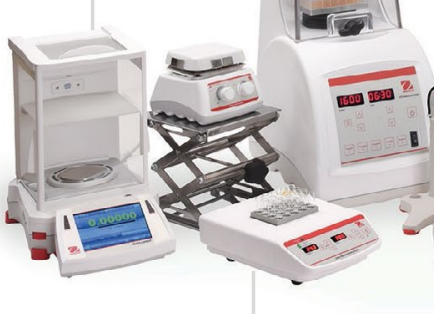

Dry Block Heater with lid features a second heater in the lid to avoid cross-contamination and minimize condensation, maintaining sample integrity.
A variety of specialized LabJaws Clamps and Lab Frames with adjustable features to provide an exact fit for labware such as thermometers, thermocouples, columns, funnels, and round bottom flasks.

\section{Extreme Environment Shake} designed for use in applications that require $\mathrm{CO}_{2}$ and humidity for optimal cell growth. Control the shaker with a remote control that sits outside the incubator, eliminating the need to open door and change incubator environment.

Quickly monitor $\mathrm{pH}$ of your sample with high-performance Benchtop Meters and Micro Electrodes idea for direct measurement in tubes or microplates.
Multi-purpose Centrifuges offering low or high-speed centrifugation platforms which can be customized to fit workflow needs using a wide variety of rotors and accessories.

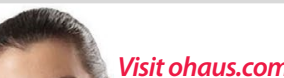
to read more about each piece of equipment 